\title{
Dynamic versus static models for photosynthesis
}

\author{
Claudia Pahl-Wostl \\ Swiss Federal Institute of Technology, Zürich, Environmental Physics, Institute for Aquatic Sciences, \\ Ueberlandstrasse 133, CH-8600 Dübendorf, Switzerland
}

Key words: photosynthesis, phytoplankton, time scales, environmental variability

\begin{abstract}
Static $\mathrm{P}(\mathrm{I})$ curves relating photosynthesis to the instantaneous light might not be adequate to describe the activity of algal cells in lakes or oceans where mixing can cause a complex pattern of light variation. In recent years experimental results have provided evidence that, subsequent to changes in light, the rate of photosynthesis may be delayed or exhibit complex temporal dynamics.

The model DYPHORA (DYnamic model for the PHOtosynthetic Rate of Algae) takes these dynamics into account by introducing two characteristic response times for the biological processes: (1) the effect of light inhibition having a time scale of minutes to a few hours and (2) the time lag of the rate of photosynthesis for increasing light intensities having a time scale of seconds to minutes.

The importance of the dynamic relative to the static description of photosynthesis depends on the time scales of the changes in the environment and the biological response, becoming significant when the time scales are comparable.
\end{abstract}

\section{Introduction}

In recent years limnologists have become more aware of the importance of the relationship between physical and biological processes. The original perspective of a lake as constituting a homogeneous environment proved to be inadequate for understanding the diversity of coexisting species. New light was shed on Hutchinsons (1963) paradox claiming an apparent violation of the principle of competitive exclusion by the advent of techniques that allow for the resolution of small-scale processes (e.g. Harris, 1980; Reynolds, 1984a, 1984b). A new interdisciplinary field of research termed 'dynamic biological limnology' (Legendre \& Demers, 1984) focusses on the variability and the coupling of biological and hydrodynamic processes.
The scale of mixing processes in the vertical dimension shows large variations from minutes to days (Denman \& Gargett, 1983; Imboden, 1989). As a consequence, algae are subject to a wide range of variation in light intensity. Under conditions of strong mixing the light climate as experienced by algae might change from bright surface light to complete darkness within minutes. The response of the rate of photosynthesis subsequent to changes in light intensity is characterized by delays and complex temporal dynamics. This was revealed in a variety of experiments and field studies (e.g. Harris \& Piccinin, 1977; Marra, 1978a, 1978b, 1980; Côté \& Platt, 1983; Vincent, Neale \& Richerson, 1984).

The majority of the mathematical models for photosynthesis, however, do not take these dynamic aspects into account. They are static de- 
scriptions of a fixed relationship between the rate of photosynthesis and the light intensity. Models of this type are not adequate to describe the response of photosynthesis under variable light conditions.

A dynamic model takes into account the typical response characteristics of the algal cell. There have been different suggestions concerning how to incorporate these effects in mathematical models (Platt \& Gallegos, 1980; Neale \& Marra, 1985; Denman \& Marra, 1986; Lande \& Lewis, 1989). DYPHORA (DYnamic model for the PHOtosynthetic Rate of Algae), the model presented here, is an attempt to find the simplest mathematical expression which is able to describe the dynamics observed in P/I-relationships. It does not aim to provide an adequate description of all physiological and biochemical processes responsible for the rate of photosynthesis.

The biologist is offered a simple tool that allows for quantitative comparison between the dynamic and the static models. A detailed description of the model and its application to simulate experimental data was published elsewhere (PahlWostl \& Imboden, 1990). In the following discussion, the focus is on the difference between the static and the dynamic type of models.

\section{Model description}

The basic idea of the DYPHORA model lies in the dynamic response of the rate of photosynthesis $P(\mathrm{t})$ to changing light intensities $I(\mathrm{t})$. As a consequence, $P$ does not simply depend on the actual instantaneous light, $I$, as is the case in most existing models, but also on the prior light history. Obviously, every dynamic model embraces a static model as well, which can be derived by keeping the driving variable $(I)$ constant long enough in order for the dependent variable $(P)$ to reach equilibrium (steady state), $P_{\text {eq }}$. Although steady state conditions hardly ever occur in nature, we first discuss the equilibrium properties of the model, thus facilitating a comparison with existing static models.

The equilibrium P/I-relationship consists of the non-inhibited tanh-function suggested by Jassby \& Platt (1976), and an equilibrium inhibition function $\Psi_{\text {eq }}(I)$, The tanh-function was chosen among various other mathematical expressions, because it proved to be the most suitable to simulate experimental data (cf. Pahl-Wostl \& Imboden, 1990). In order to simplify the discussion, rates of photosynthesis (P) will be expressed in relative units. All variables and their standard units are listed in Table 1.

The parameter $P_{\max }$ of Jassby \& Platt (1976) is used as a scaling factor:

$$
P_{\mathrm{eq}}^{*}(I)=\frac{\hat{P}_{\mathrm{eq}}}{\hat{P}_{\mathrm{max}}}=\tanh \left(I / I_{k}\right),
$$

where $\hat{P}_{\max }$ and $\hat{P}_{\text {eq }}$ are the rates of photosynthesis with their corresponding units and $P_{\text {eq }}^{*}$ is the non-inhibited, dimensionless rate.

The equilibrium inhibition function $\Psi_{\mathrm{eq}}(I)$ is

$$
\Psi_{\text {eq }}(I)=\left\{\begin{array}{ll}
0 & \text { for } I \leq I_{\text {crit }} \\
\left(I-I_{\text {crit }}\right) K \tau_{\iota} & \text { for } I>I_{\text {crit }}
\end{array},\right.
$$

where $I_{\text {crit }}$ is the critical light intensity for the onset of photo-inhibition. The meaning of the (constant) parameters $K$ and $\tau_{\mathrm{i}}$ will become clear when we discuss the dynamic properties of the model.

The inhibited equilibrium rate (for $I>I_{\text {crit }}$ ) is defined as

$$
\begin{aligned}
P_{\mathrm{eq}}(I) & =\frac{P_{\mathrm{eq}}^{*}(I)}{1+\Psi_{\mathrm{eq}}(I)} \\
& =\frac{\tanh \left(I / I_{k}\right)}{1+\left(I-I_{\mathrm{crit}}\right) K \tau_{i}}
\end{aligned}
$$

The dynamic aspects of photoresponse are brought into DYPHORA in two ways. First, it is postulated that for rising light intensity, $I$, the non-inhibited rate of photosynthesis needs some time to adjust itself to the new light level. The adjustment is described by the response time $\tau_{\mathrm{r}}$. In contrast, for decreasing $I$ P drops back to its lower value without a time delay. Mathematically, this behaviour can be described by the differential equation 


$$
\frac{d P^{*}}{d t}=-k_{r}\left(P^{*}-P_{\mathrm{eq}}^{*}\right),
$$

where

$$
k_{r}=\left\{\begin{array}{ll}
1 / \tau_{r} & \text { for } P^{*} \leq P_{\mathrm{eq}}^{*} \\
\infty & \text { for } P^{*}>P_{\mathrm{eq}}^{*}
\end{array} .\right.
$$

$P^{*}$ is the rate of photosynthesis, i.e. if photoinhibition did not exist. Eq. (4) expresses the fact that $P^{*}$ is always changing in the direction of the non-inhibited rate $P_{\mathrm{eq}}^{*}$ (Eq. 1) and that the downward adjustment occurs instantaneously.

The second dynamic effect is related to the inhibition function, $\Psi$, by assuming that the effect of supracritical light decays with some time constant $\tau_{\mathrm{i}}$. Thus, $\Psi$ is described by the linear differential equation (see Table 1 for definitions):

$$
\frac{d \Psi}{d t}=K I_{\mathrm{ex}}-\frac{1}{\tau_{i}} \Psi,
$$

where the first term on the right-hand side describes the 'production' of inhibition and the second term describes the linear decay of accumulated inhibition. For constant $I, \Psi$ reaches a steady state $(d \Psi / d t=0)$ which is either $0\left(I \leq I_{\text {crit }}\right)$ or $\Psi_{\text {eq }}=K\left(I-I_{\text {crit }}\right) \tau_{\mathrm{i}}$, in accordance with Eq. (2). For time-variable light, $\Psi(t)$ is given by the convolution integral

$$
\begin{aligned}
\Psi(t)= & K \int_{0}^{t} I_{\mathrm{ex}}\left(t^{\prime}\right) \exp \frac{\left(t^{\prime}-t\right)}{\tau_{i}} d t^{\prime} \\
& +\Psi_{0} \mathrm{e}^{-t / \tau_{i}},
\end{aligned}
$$

where $\Psi_{0}$ is the inhibition function at $t=0$.

Having described the two dynamic elements we can now easily calculate the actual rate of photosynthesis, $P(\mathrm{t})$, by the equation

$$
P(t)=\frac{P^{*}(t)}{1+\Psi(t)} .
$$

The dynamic behaviour of $P(t)$ is controlled by two intrinsic time scales, the response time for increasing light $\tau_{\mathrm{r}}$, and the inhibition decay time

\begin{tabular}{|c|c|c|}
\hline Symbol & Units & Definition \\
\hline$t$ & S & Time \\
\hline$I$ & $\mu \mathrm{E} \mathrm{m}^{-2} \mathrm{~s}^{-1}$ & Light intensity \\
\hline$I_{\mathrm{k}}{ }^{I}$ & $\mu \mathrm{E} \mathrm{m}^{-2} \mathrm{~s}^{-1}$ & Characteristic light intensity describing the equilibrium productivity curve \\
\hline$I_{\text {crit }}$ & $\mu \mathrm{E} \mathrm{m}^{-2} \mathrm{~s}^{-1}$ & Critical light intensity for onset of light inhibition \\
\hline$I_{\mathrm{ex}}= \begin{cases}0 & I \leq I_{\text {crit }}\end{cases}$ & 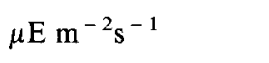 & Inhibiting excess light intensity \\
\hline$P^{*} \quad\left\{I-I_{\text {crit }} I>I_{\text {crit }}\right.$ & Note $^{2}$ & (Potential) rate of photosynthesis without photoinhibition \\
\hline$P$ & $\operatorname{Note}^{2}$ & Actual rate of photosynthesis \\
\hline$P_{\mathrm{eq}}^{*}$ & Note $^{2}$ & Equilibrium rate of photosynthesis without inhibition (eq. 1) \\
\hline$P_{\text {eq }}$ & Note $^{2}$ & Equilibrium rate of photosynthesis with inhibition (eq. 3) \\
\hline$\tau_{\mathrm{r}}$ & s & Response time of $P^{*}$ for increasing I \\
\hline$\Psi$ & dimensionless & Light inhibition function \\
\hline$\Psi_{\mathrm{eq}}$ & dimensionless & Equilibrium inhibition function for constant $I$ \\
\hline$K$ & $\left(\mu \mathrm{E} \mathrm{m}^{-2} \mathrm{~s}^{-1}\right)^{-1} \mathrm{~s}^{-1}$ & Inhibition growth constant \\
\hline$\tau_{\mathrm{i}}$ & s & Inhibition decay time \\
\hline
\end{tabular}
$\tau_{\mathbf{i}}$

\section{Results and discussion}

In the following discussion, the expression 'static model' refers to calculation of P using the steady

Table 1. Definition of DYPHORA variables and their standard units.

${ }^{1} I_{k}$ is related to the equilibrium photosynthesis function proposed by Jassby \& Platt $(1976), \hat{P}_{\mathrm{eq}}=\hat{P}_{\max } \tanh \left(\alpha I / \hat{P}_{\max }\right): I_{\mathrm{k}}=\hat{P}_{\max } / \alpha$.

2 All rates of photosynthesis are expressed relative to the maximum equilibrium rate of Jassby \& Platt (1976), $\hat{P}_{\text {max }}$, and are thus dimensionless (see eq. 1). 
state relationship (Eq. 3). This implies that the rate of photosynthesis is always in equilibrium with the actual light intensity no matter its rate of change. The expression 'dynamic model' refers to calculations of $P$ using Eq. (7), where the rate of photosynthesis shows a dynamic response to changes in light intensity. The essential difference between these descriptions is exemplified in Fig. 1. The dynamic and the static model have been used to simulate the response of the rate of photosynthesis to sudden changes in light intensity. As shown in Fig. 1a, the light intensity was assumed to jump at $t=250$ from 0 to a light intensity (200) below the threshold for inhibition. The static model follows the light change without delay, whereas the dynamic model shows a time lag which is characterized by the response time $\tau_{\mathrm{r}}$. At $t=750$ the light intensity was assumed to jump to a value exceeding the threshold for inhibition. The static model predicts an immediate drop of
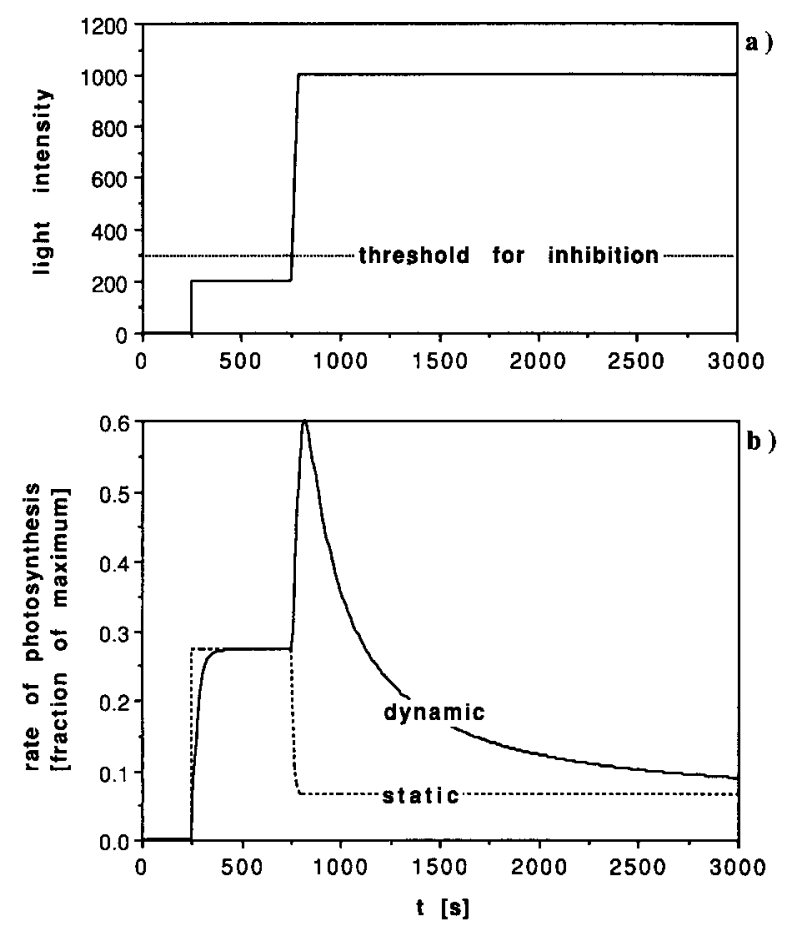

Fig. 1. (a) Step function of light intensity. (b) Comparison of the response of the rate of photosynthesis as calculated using dynamic and the static models. Parameter values: $\tau_{\mathrm{j}}=1800$, $\tau_{\mathrm{r}}=30 \mathrm{~s} ; \quad I_{\text {crit }}=300, \quad I_{\mathrm{k}}=714 \mu \mathrm{E} \mathrm{m}^{-2} \mathrm{~s}^{-1} ; \quad K 2.010^{-7}$ $\left(\mu \mathrm{E} \mathrm{m}^{-2} \mathrm{~s}^{-1}\right)^{-1} \mathrm{~s}^{-1}$.
$\mathrm{P}$ to its fully inhibited value. The dynamic model however, shows an initial stimulation of $\mathrm{P}$, until the effect of inhibition sets in on a time scale characterized by the decay time of the inhibition $\tau_{i}$, which occurs more slowly than according to the static model. This type of behaviour has been found in a variety of experiments (e.g. Harris \& Piccinin, 1977; Marra, 1978a, b). In fact, this kind of observation motivated the introduction of two time scales into DYPHORA (Pahl-Wostl \& Imboden, 1990).

The time scale for inhibition proved to be quite variable. We estimated the values for $\tau_{\mathrm{i}}$ from published experimental data and found values ranging from 10 to 15 minutes up to a few hours (Pahl-Wostl \& Imboden, 1990).

\section{Diurnal time course of photosynthesis}

\section{A. Without mixing}

An example for the effect of inhibition on a time scale of hours is what has been called 'afternoon depression'. The diurnal time course of the light intensity is shown in Fig. 2a. The day starts at $t=0(\mathrm{~h})$ and ends at $t=12 \mathrm{~h}$. The corresponding rates of photosynthesis are represented in Fig. $2 \mathrm{~b}$. The scattered data were obtained in laboratory experiments by Marra (1978a) and the solid curve in simulations with the dynamic model of DYPHORA. In the afternoon the photosynthesis at a given light intensity proves to be considerably lower than for the same light intensity in the morning. This effect becomes even more obvious in a graphic representation of the $P(I)$ curves for the morning and the afternoon. As shown in Fig. 2c a typical hysteresis is obtained, which is characteristic of a system exhibiting a memory effect. The hysteresis effect depends on the rate of change of the light and the response characteristics of the cell. This can be demonstrated in a model 'experiment' where the light intensity is varied with a constant rate from 0 to a maximum and then back to zero again. The $P(I)$ curves as obtained for three different rates of light variation are shown in Fig. 3. The arrows indicate whether 

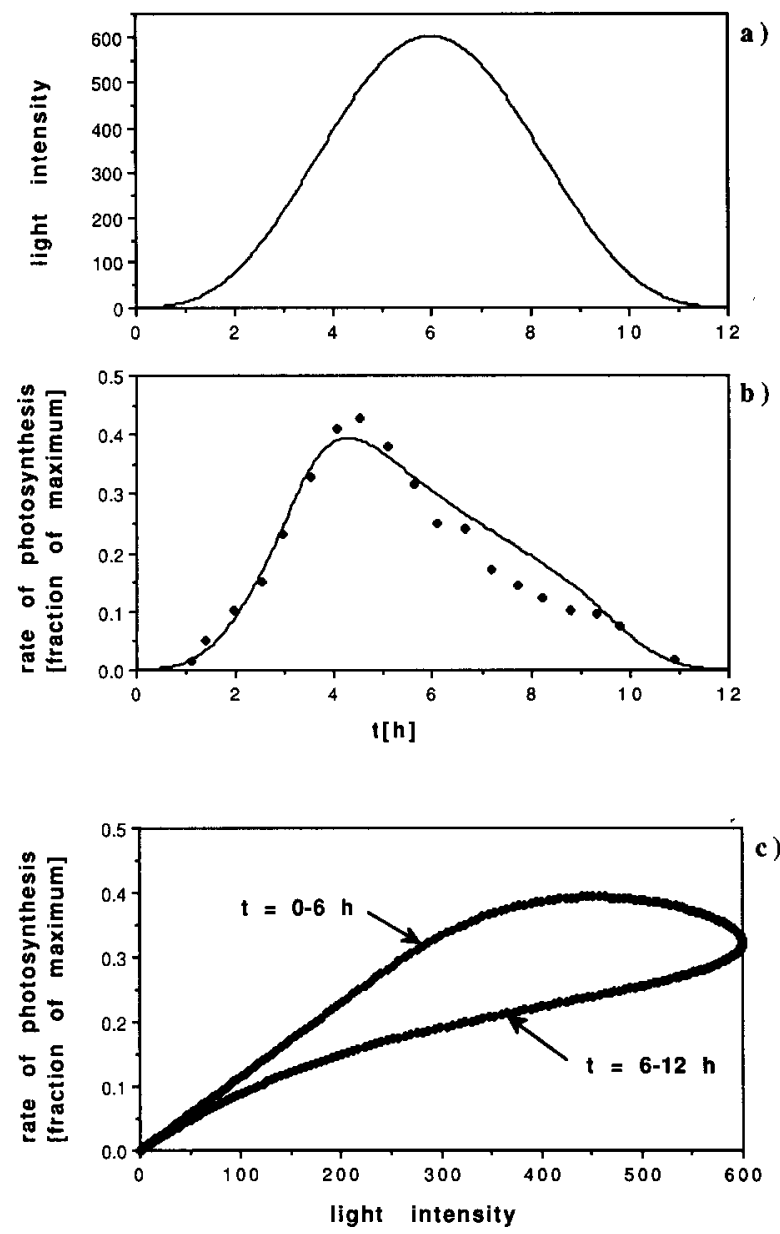

Fig. 2. a) Time course (h) of light intensity $\left(\mu \mathrm{E} \mathrm{m}^{-2} \mathrm{~s}^{-1}\right)$. b) Rate of photosynthesis as obtained for light variation shown in $2 \mathrm{a}$. Solid curve represents results from simulations and scattered points represent results from experiments by Marra (1978a). c) P(I) curve derived from b. Parameter values: $\tau_{\mathrm{i}}=5400, \quad \tau_{\mathrm{r}}=30 \mathrm{~s} ; \quad I_{\text {crit }}=250, \quad I_{\mathrm{k}}=825 \mu \mathrm{E} \mathrm{m}^{-2} \mathrm{~s}^{-1}$; $\left.K=7.410^{-7} \mu \mathrm{E} \mathrm{m}^{-2} \mathrm{~s}^{-1}\right)^{-1} \mathrm{~s}^{-1}$.

the curve was obtained for increasing or decreasing light intensities.

The time $\tau_{\mathrm{p}}$ it takes for the light to increase from $I_{\text {crit }}$ to 10000 is $2.5,25$ and $250 \mathrm{~min}$ for the rates of $5,0.5$ and $0.05\left(\mu \mathrm{E} \mathrm{m}^{-2} \mathrm{~s}^{-1}\right) \mathrm{s}^{-1}$, respectively.

The time scales $\tau_{\mathrm{p}}$ for physical change have to be compared to the time scales of biological response characterized by $\tau_{\mathrm{r}}=0.5 \mathrm{~min}$, i.e. response time for increasing light intensities, and $\tau_{\mathrm{i}}=30 \mathrm{~min}$, i.e. response time for photo-inhibition.

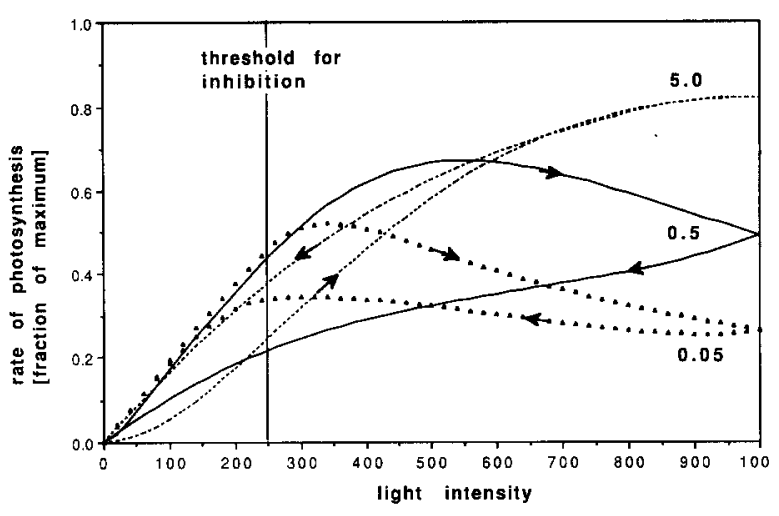

Fig. 3. Hysteresis of $P$ for linearly increasing and decreasing light intensity $\left(\mu \mathrm{E} \mathrm{m}^{-2} \mathrm{~s}^{-1}\right)$. The rate of change of light intensity is shown in $\left.\mu \mathrm{E} \mathrm{m}^{2} \mathrm{~s}^{-1}\right) \mathrm{s}^{-1}$. The arrows indicate the direction of light change. Parameter values: $\tau_{\mathrm{i}}=1800, \tau_{\mathrm{r}}=30 \mathrm{~s}$; $\left.I_{\text {crit }}=250, I_{\mathrm{k}}=500 \mu \mathrm{E} \mathrm{m}^{-2} \mathrm{~s}^{-1} ; K=2.210^{-6} \mu \mathrm{m}^{-2} \mathrm{~s}^{-1}\right)^{-1}$ $\mathbf{s}^{-1}$.

Case (1): rate $=5.0 ; \tau_{\mathrm{p}} \approx \tau_{\mathrm{r}}$ and $\tau_{\mathrm{p}}<<\tau_{\mathrm{i}}$. No effect of photo-inhibition is observed. However, due to the time lag of the rate of photosynthesis, higher rates are observed for the decreasing light intensities than for the increasing light intensities.

Case (2): rate $=0.5 ; \tau_{\mathrm{p}}>\tau_{\mathrm{r}}$ and $\tau_{\mathrm{p}} \approx \tau_{\mathrm{i}}$. The effect of hysteresis due to photo-inhibition is very pronounced. Much higher rates are obtained for increasing than for decreasing light intensities.

Case (3): rate $=0.05 ; \tau_{p}>>\tau_{r}$ and $\tau_{p}>\tau_{i}$. Due to the slow changes in the physical environment the rate of photosynthesis is close to equilibrium with the instantaneous light intensities and the effect of hysteresis is less pronounced.

Whenever the rates of change in the physical environment are comparable to the rates of the biological response we observe pronounced effects of hysteresis which cannot be explained with a static model.

\section{B. With mixing}

In the above cases we assumed that the algae remain at a fixed spatial location and that the only source of variability is provided by the diurnal time course of light intensity. However, if we allow for mixing, different effects contribute to the vari- 
ability of the light during a day as summarized in Fig. 4.

The first physical time scale is determined by the length of the day and the variation of the surface light intensity $I_{0}$, which can be conveniently expressed by a cubic sine function (Marra, 1978a):

$$
\mathrm{I}_{0}(t)=I_{\max } \sin ^{3}(\pi t / L d)
$$

where $L d=$ length of the day ( $12 \mathrm{~h})$.

The second is the time scale of mixing. Mixing is expressed in a simplified description by a circular motion, which leads to a sine-shaped variation of the depth $z$ of the moving algae:

$$
\mathrm{z}(\mathrm{t})=\mathrm{z}_{\text {mix }}\{1+\sin (2 \pi t / L p)\}
$$

where $L p=$ length of the rotation period $(60 \mathrm{~min})$,

and

$z_{\text {mix }}=$ depth of the mixed layer $(10 \mathrm{~m})$.

Hence the time scale of mixing is equal to the length of the rotation period. The light intensity $I_{z}$

\section{DAILY LIGHT ENVIRONMENT OF ALGAE}
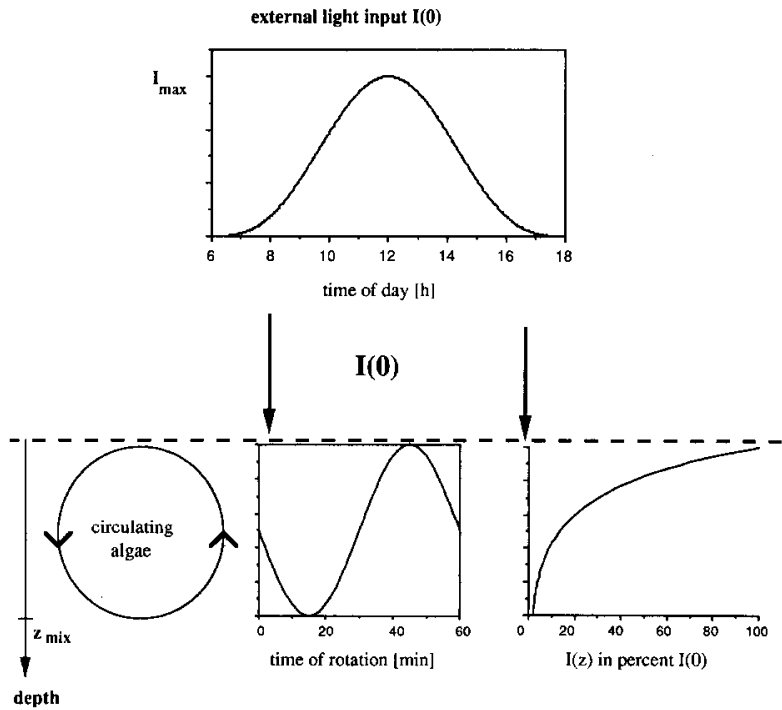

Fig. 4. Factors that determine the light climate experienced by algae if mixing is assumed to be equivalent to a circular motion $\left(z_{\mathrm{mix}}=10 \mathrm{~m} ; \varepsilon=0.3 \mathrm{~m}^{-1}\right.$; see text). The dashed line represents the surface of the water body. as experienced by the moving particle can be expressed as:

$$
I_{z}(t)=I_{0}(t) \mathrm{e}^{-\varepsilon z(t)}
$$

where $\varepsilon$ is the light extinction coefficient $\left(0.3 \mathrm{~m}^{-1}\right)$.

The resulting light climate as experienced by the moving algae during a day is shown in Fig. 5a. This variation in light intensity was used as input for the model simulations of the diurnal time course of the rate of photosynthesis under mixed conditions. The results obtained with the dynamic and the static model are shown in Fig. 5b. The values of the parameters are based on results obtained from experiments of Marra (1978a, b). For a comparison of results from model simulation
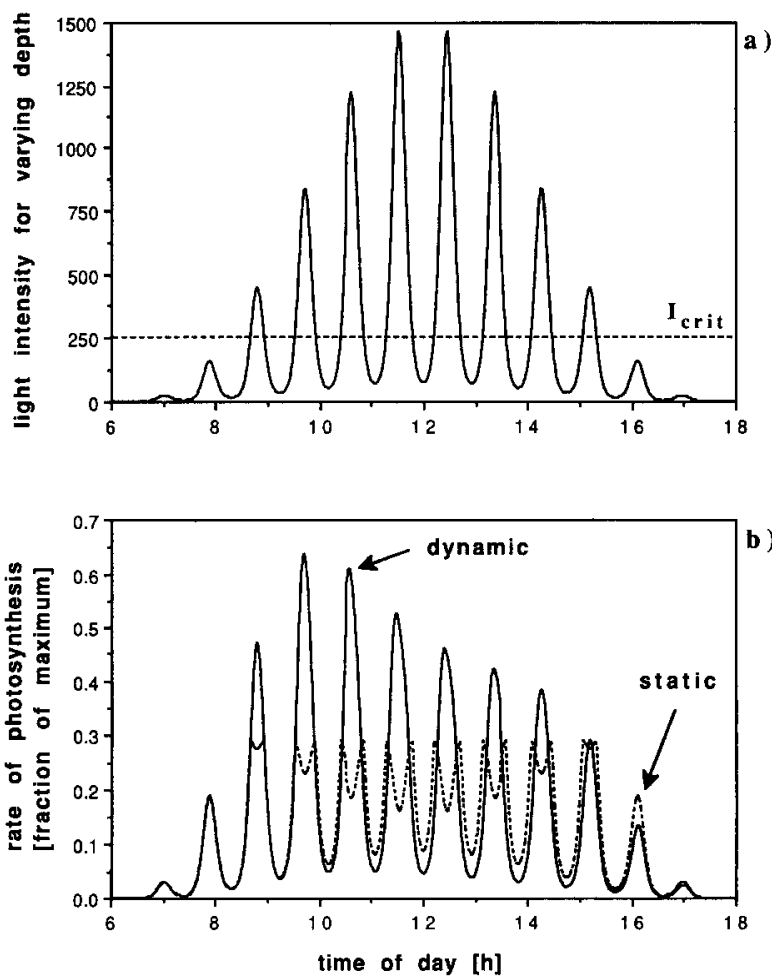

Fig. 5. a) Light climate experienced by algae for varying external factors as described in Fig. 4. b) Diurnal time course of photosynthesis as obtained with the dynamic (solid curve) and the static (dashed curve) model based on the variation of light intensity under mixing conditions as shown in Fig. 5a $\left(I_{\max }=1500 \mu \mathrm{E} \mathrm{m}^{-2} \mathrm{~s}^{-1}\right)$. Parameter values as for Fig. 2 . 
with experimental data of Marra (1978a, b), see Pahl-Wostl \& Imboden (1990).

In the static model $\mathrm{P}$ tracks the light variation. This leads to an over-estimation of the effect of inhibition. In the dynamic model inhibition sets in slowly, depending on the response time $\tau_{i}$, but recovery takes some time as well, as becomes obvious during the late afternoon hours. For these hours the static model assumes immediate recovery and yields higher values of $P$. The importance of the different effects may change depending on the chosen conditions. To investigate the influence of the ratio of physical and biological time scale, the simulations were repeated for a range of values for the mixing period. The mean rate of photosynthesis averaged over one day as obtained with the dynamic model is expressed as a percent age of the corresponding result obtained with the static model (Fig. 6). Curve II represents the results based on the same parameter set used in Fig. $5 \mathrm{~b}$. The static model underestimates the rate under variable light conditions. This is caused by the assumption of an immediate onset of inhibition once the light exceeds the critical threshold.

To investigate the influence of the response to increasing light, the simulations were repeated with a value of $\tau_{r}$ four times higher, i.e. the response is slower than the one used to obtain cur-

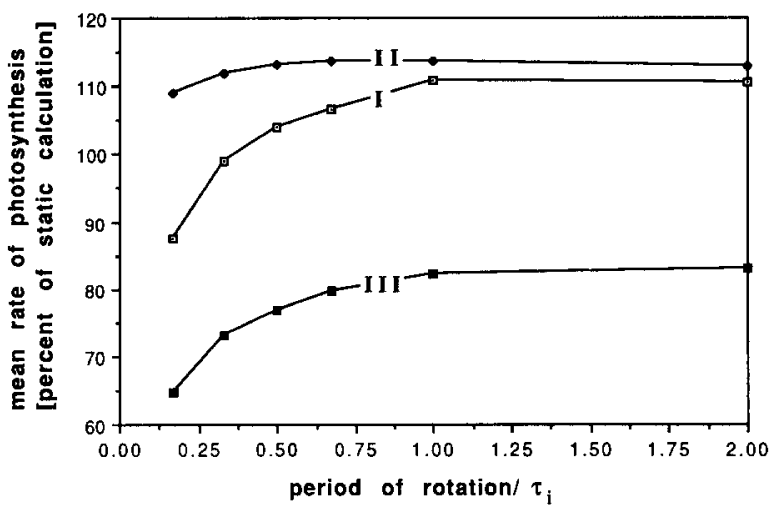

Fig. 6. Mean rate of photosynthesis averaged over one day obtained with the dynamic model (expressed as percentage of the rate obtained with the static model) as a function of the ratio of the time scale of mixing and the biological decay time for inhibition $\tau_{\mathbf{i}}$. Parameter values as in Fig. 2, except for $\tau_{\mathrm{r}}=120 \mathrm{~s}$ and $K=3.710^{-6}\left(\mu \mathrm{E} \mathrm{m}^{-2} \mathrm{~s}^{-1}\right)^{-1} \mathrm{~s}^{-1}$. ve II (Fig. 6). The results are represented by curve I. Now the static model gives a higher rate of photosynthesis for fast changes in light intensity, where in the dynamic description the rate of photosynthesis lags behind the equilibrium rate. However, for slow mixing the effects of the inhibition prevail and results are similar as for II.

Under certain conditions both models produce the same value of $P$, when averaged over the day. This does not imply that the static model describes the phenomenon accurately, but rather that the various effects cancel each other.

The influence of the sensitivity to inhibition was tested by increasing the inhibition growth constant, $K$, five-fold. The results are represented by curve III. Now the situation is reversed. The static model severely overestimates the rate, because it implies immediate recovery from damaging light conditions once the light passes below the critical threshold. The dynamic model, however, preserves a 'memory' for the damaging influence of the high light intensities.

We conclude that the difference between the static and dynamic model is critically dependent on the ratio of the biological and physical time scales and on the sensitivity to inhibition.

\section{Conclusions}

It is not possible to arrive at simple recommendations concerning the application of the dynamic versus the static model. From a physiological point of view it is reasonable to assume that the inhibitory effect of high light intensities depends on the length of exposure. It is known that phytoplankton show a variety of physiological responses on different time scales subsequent to changing light conditions (e.g. Vincent, 1990). In all situations where the time scale of the biological response and the environmental changes are in the same order of magnitude, steady state models are not adequate. Further experimental investigations are necessary to learn more about biological characteristics in relation to changes in the physical environment. We hope that the simple modelling approach presented here stimulates in- 
terest from biologists to apply dynamic models to explain their experimental findings.

\section{References}

Côté, B. \& T. Platt, 1983. Day-to-day variations in the springsummer photosynthetic parameters of coastal marine phytoplankton. Limnol. Oceanogr. 28: 320-344.

Denman, K. L. \& A. E. Gargett, 1983. Time and space scales of vertical mixing and advection of phytoplankton in the upper ocean. Limnol. Oceanogr. 28: 807-815.

Denman, K. L. \& J. Marra, 1986. Modelling the time dependent photoadaptation of phytoplankton to fluctuating light. In L. C. Nihoul (ed.), Marine interfaces ecohydrodynamics. Elsevier Oceanogr. Series 42: 341-360.

Harris, G. P., 1980. Temporal and spatial scales in phytoplankton ecology. Mechanisms, methods, models and management. Can. J. Fish. aquat. Sci. 37: 877-900.

Harris, G. P. \& B. B. Piccinin, 1977. Photosynthesis by natural phytoplankton populations. Arch. Hydrobiol. 80: 405456.

Hutchinson, G. E., 1961. The paradox of the plankton. Am. Nat. 95: 137-146.

Imboden, D. M., 1990. Mixing and transport in lakes: Mechanisms and ecological reference. In M. Tilzer \& C. Serruya (eds), Ecological Structure and Function of Large Lakes. Science Tech. Publishers, Madison, Wisconsin.

Jassby, A. D. \& T. Platt, 1976. Mathematical formulation of the relationship between photosynthesis and light for phytoplankton. Limnol. Oceanogr. 21: 540-547.

Lande, R. \& M. Lewis, 1989. Models of photoadaptation by algal cells in a turbulent mixed layer. Deep Sea Res. 36: 1161-1175.
Legendre, L. \& S. Demers, 1984. Towards dynamic biological oceanography and limnology. Can. J. Fish. aquat. Sci. 41: 2-19.

Marra, J., 1978a. Effect of short-term variations in light intensity on photosynthesis of a marine phytoplankter: a laboratory simulation study. Mar. Biol. 46: 191-202.

Marra, J., 1978b. Phytoplankton photosynthesis response to vertical movement in a mixed layer. Mar. Biol. 46: 203-208.

Marra, J., 1980. Vertical mixing and primary production. In P. G. Falkowski (ed.), Primary productivity in the sea. Plenum Press, NY \& London: 121-137.

Neale, P. J. \& J. Marra, 1985. Short-term variation of $P_{\max }$ under natural irradiance conditions: a model and its implications. Mar. Ecol. Prog. Ser. 26: 113-124.

Pahl-Wostl, C. \& D. Imboden, 1990. DYPHORA - A dynamic model for the rate of photosynthesis of algae. $J$. Plankton Res, 12: 1207-1221.

Platt, T. \& C. L. Gallegos, 1980. Modelling primary production. In: P. G. Falkowski (ed.), Primary productivity in the sea. Plenum Press, NY \& London: 339-362.

Reynolds, C. S., 1984a. The ecology of freshwater phytoplankton. Cambridge University Press, Cambridge.

Reynolds, C. S., 1984b. Phytoplankton periodicity: the interaction of form, function and environmental variability. Freshwat. Biol. 14: 11-142.

Vincent, W. F., 1990. The dynamic coupling between photosynthesis and light in the phytoplankton environment. Verh. int. Ver. Limnol. 24: 25-37.

Vincent, W. F., P. J. Neale \& P. J. Richerson, 1984. Photoinhibition: algal responses to bright light during diel stratification and mixing in a tropical alpine lake. J. Phycol. 20: 201-211. 